\title{
COVID-19 IN AUTOIMMUNE RHEUMATIC DISEASES - FREQUENCY, COURSE, EFFECTIVENESS AND COMPLICATIONS IN VACCINATION
}

\author{
Z. Dimova, V. Boyadzhieva, N. Stoilov, S. Monov, R. Stoilov \\ Clinic of Rheumatology, University Hospital "Sv. Ivan Rilski”, MU - Sofia, Bulgaria
}

\begin{abstract}
The onset of the pandemic caused by the new coronavirus has raised many questions in the global rheumatology society regarding the clinical course of the disease, effectiveness and complications of SARS-SoV-2 vaccination. The aim of this review is to analyze the information available up to date on the course of COVID-19 in patients with autoimmune rheumatic diseases (ARD), the frequency of complications in these patients, the effectiveness and side effects of vaccination against COVID-19. Various studies in Europe and the United States have found that the risk of severe COVID-19 in patients with $A R D$ is similar to that in the general population. Negative prognostic factors are thought to be old age, comorbidity, moderate/high ARD activity, and corticosteroid (CS) intake at doses > $10 \mathrm{mg} /$ day. Patients with systemic ARD are considered to be at higher risk of thromboembolic events due to chronic inflammation and/or the presence of antiphospholipid syndrome, as well as the nature of COVID-19an endothelial lesion causing thrombosis and microangiopathy. It is observed a higher risk of severe pneumonia in patients treated with Rituximab, as well as an increase in hospitalizations and deaths amongst them. The opposite trend is observed in patients treated with anti-TNF. With the development of vaccines against COVID-19, questions arise about their effectiveness in patients with ARD, as well as the frequency of complications associated with them. In these studies, slightly lower levels of neutralizing antibodies were observed in patients with ARD compared to the general population, most commonly associated with Methotrexate or targeted synthetic DMARDs. The most common side effects seen in these patients after vaccination are pain at the injection site, fatigue and headache. Complaints of the musculoskeletal system are more common in patients with ARD than in the general population. Exacerbations of the underlying disease following vaccination, requiring hospitalization, are rare.
\end{abstract}

Key words: SARS-CoV-2, autoimmune rheumatic diseases; complications; vaccination

\section{INTRODUCTION}

In March 2020, the WHO declared a global pandemic following the new coronavirus, SARS-CoV-2, which causes a disease characterized by an acute respiratory distress syndrome called COVID-19. It is still unclear about patients with autoimmune rheumatic diseases and COVID-19 [1]. Of interest is the frequency and severity of the disease in this group of patients and the relative share of serious, life-threatening forms of the disease. Patients with autoimmune rheumatic diseases have an altered immune response, which makes them at risk for the virus [2]. Many of them carry out immunosuppressive therapy, which further compromises the body's immune response to external factors.

The aim of this review is to analyze data on the frequency and severity of COVID-19 infection in patients with autoimmune rheumatic diseases, complications, efficacy and adverse events following vaccination with currently registered vaccines.
Morbidity and characteristics of SARS-CoV-2 infection in patients with autoimmune inflammatory rheumatic diseases

The first data on the incidence of COVID-19 in patients with autoimmune rheumatic diseases (ARD) are from small studies in Italy, Spain and the United States. Their data show that the incidence of COVID-19 among rheumatic patients is similar to that of the normal population [2, 3, 4]. The global spread of SARS-CoV-2 and the registration of new strains of infection have necessitated more and more studies involving different therapeutic algorithms. A Swedish national study by Bower et al., conducted between March and September 2020, compared a group with inflammatory joint disease (IJD) with a population reference group (2015-2019). The risk of hospitalization $(0.5 \%$ in IJD vs. $0.3 \%$ in the reference population), intensive care $(0.04 \%$ vs. $0.03 \%)$ and death $(0.10 \%$ vs. $0.07 \%)$ due to COVID- 19 was low in patients with ARD and similar in the general population. This study summarizes that the overall 
absolute risks for patients with inflammatory joint disease are low and the level of increased risk is largely proportional to that in the general population and is explained by comorbidities. A similar risk of severe COVID-19 has been identified in both ARD and the general population, suggesting a negative prognostic effect of comorbidities [5]. The data summarized by the German registry provide more detailed information on patients with ARD. 468 patients with ARD and PCR-confirmed SARS-CoV-2 infection were studied. Patients were divided into three groups, and the need for hospitalization of patients was used as an indicator of severe course. Patients are divided into several groups: (1) non-hospitalized patients, (2) hospitalized patients without the need for invasive ventilation, (3) hospitalized patients with the need for invasive ventilation. In this cohort, the most common disease is RA, which affects 225 patients (48\%) and is associated with a high hospitalization rate. Of these, 62 are hospitalized and 17 need invasive ventilation. Of the 140 patients with SpA and PCR-confirmed SARS-CoV-2 infection, 20 were hospitalized and 5 required invasive ventilation [6]. In this cohort, compared to RA, patients with SpA showed a lower risk of hospitalization (OR 0.46; 95\% $\mathrm{Cl}$ 0.23-0.91). Of the group of ARD patients, only $38 \%$ had no other chronic diseases. The most common is high blood pressure, followed by obesity, cardiovascular disease (CVD) and diabetes. Regarding the administered therapy for ARD, 193 patients were treated with monotherapy with conventional synthetic disease-modifying antirheumatic drugs (csDMARDs), 167 patients with biological DMARDs and 145 patients with corticosteroids (CS), at a dose of $\leq 5 \mathrm{mg} /$ day. Older patients with significant comorbidities, moderate to high ARD activity, and CS therapy $>5 \mathrm{mg} /$ day are more likely to be hospitalized [6]. In 2020, a clinical study was conducted in the United States, including 2379 patients with systemic ARD and COVID-19 and a control group of 142,750 patients with COVID-19 who did not have systemic ARD. The results of the study showed that patients with autoimmune rheumatic disease with COVID-19 infection had a higher risk of hospitalization, intensive care, acute renal failure and venous thromboembolism than the control group. The authors found that rheumatic patients had similar mortality compared to the general population. A significantly higher risk of venous thromboembolism has been observed in patients with systemic ARD [7].

COVID-19 is associated with severe endothelial damage, which is involved in the process of thrombosis and microangiopathy [8]. Patients with sys- temic ARD are at higher risk of thromboembolism due to chronic inflammation and/or the presence of antiphospholipid syndrome. In another comparative study in the United States, there was a $40 \%$ higher risk of venous thromboembolism in patients with rheumatoid arthritis (RA) than in the general population [9]. Interesting data are presented by a study of patients with autoimmune rheumatic diseases in France, which found that mortality associated with COVID-19 is associated with major risk factors (age, male gender and some diseases) and disease-specific factors (disease activity and medications taken). Moderate or high disease activity has been shown to be a risk factor for more severe disease. The French study emphasizes the importance of adequate control of ARD activity with DMARDs without high maintenance doses of corticosteroids. In a large proportion of rheumatic patients, glucocorticoids are the mainstay of treatment. Gianfrancesco and colleagues found that basic therapy with Prednisolone $>10 \mathrm{mg} /$ day increased the risk of developing severe COVID-19 and the need for hospitalization [11] Treatment with Tumor Necrotizing Factor (anti-TNF) inhibitors is associated with a reduced incidence of hospitalizations, while antimalarial therapy is not associated with a change in the incidence of hospitalizations for COVID-19 [11, 12, 13].

A large study in Spain examined the risk of developing severe COVID-19 in patients with autoimmune rheumatic diseases. 4592 patients diagnosed with ARD were analyzed, 883 of whom were treated with biologic therapy or JAK-inhibitor. During the study, 2,315 patients were admitted to the hospital with SARS-CoV-2 infection, of which only 41 (1.8\%) had autoimmune rheumatic disease. All patients were diagnosed with pneumonia and respiratory failure. In the study group there is a higher proportion of patients with Sjögren's syndrome, vasculitis and systemic lupus erythematosus. An increased risk of hospitalization and death has been reported in patients treated with Rituximab. Patients with ARD, especially those treated with RTX, are at higher risk of severe pneumonia due to SARS-CoV-2 compared to the general population. The opposite trend is observed with anti-TNF therapy [14].

A few months after the registration of the first patients, a national registry was established for patients infected with SARS-CoV2 with ARD in Germany. 104 patients with autoimmune diseases infected with SARS-CoV-2 were followed. Among them, 45\% have RA, 59\% have at least 1 concomitant disease and $42 \%$ are on therapy with bDMARDs, and $32 \%$ require hospitalization. The results of the study show 
that patients with ARD have a higher risk of infection than the general population. Important factors remain the activity of the underlying disease, concomitant diseases and the dosage regimen of immunosuppressive therapy. The registry allows for the rapid generation of data in this emerging situation and for a better understanding of the course of SARS-CoV2 infection in patients with ARD, with a particular focus on their concomitant treatment. The results of the studies provide essential information to physicians about patients with ARD and support the development of recommendations for patients with ARD during this pandemic [15]. The Brazilian registry (ReumaCoV Brasil register) includes 334 patients with ARD and COVID-19. The largest percentage of them were diagnosed with RA (28.4\%), with the most common therapies being Hydroxychloroquine (34.9\%), glucocorticosteroids (34.2\%), MTX $(20.1 \%)$ and anti-TNF (22.2\%). The results of the study show that age, corticosteroid use, and cyclophosphamide are associated with adverse outcomes of SARS-CoV-2 infection, whereas anti-TNF therapy has a protective role in the development of severe COVID-19 [16].

Table 1 presents small studies confirming these data [17).

\section{Efficacy and complications of vaccination} against COVID-19

To date, the COVID-19 pandemic has affected more than 194 million people and has killed nearly 4.2 million people [19]. Currently, the only solution

Table 1. Main studies focused on the frequency, results and prognostic factors of COVID-19 in patients with rheumatoid arthritis and spondyloarthritis**

\begin{tabular}{|c|c|c|c|c|c|c|c|}
\hline \multirow{2}{*}{ Clinical trial } & \multirow{2}{*}{ Design } & \multirow{2}{*}{$\begin{array}{l}\text { Number of } \\
\text { patients }\end{array}$} & \multicolumn{3}{|c|}{ Type of ARD } & \multirow{2}{*}{ Exit from COVID-19 } & \multirow{2}{*}{ Prognostic factors } \\
\hline & & & RA & SpA & PsA & & \\
\hline $\begin{array}{l}\text { FAI2R /SFR/SNFMI/ } \\
\text { SOFREMIP/CRI/IMIDIATE } \\
\text { consortium and contributors }\end{array}$ & 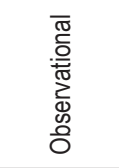 & 694 & 213 & 165 & 70 & $\begin{array}{l}\text { Bad COVID-19/ } \\
\text { hospitalisations in RA: 29; } \\
\text { SpA: 5; PsA: } 6\end{array}$ & $\begin{array}{l}\text { Older age } \\
\text { Accompanying diseases } \\
\text { Prolonged use of steroids }\end{array}$ \\
\hline Gianfrancesco et al. & 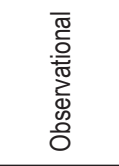 & 600 & 225 & 48 & 74 & $\begin{array}{l}\text { Bad COVID-19/ } \\
\text { hospitalisations in RA: } \\
\text { 104; SpA: 16; PsA: } 22\end{array}$ & $\begin{array}{l}\text { Older age } \\
\text { Accompanying diseases } \\
\text { Higher dose of steroids }\end{array}$ \\
\hline Hasseli et al. & 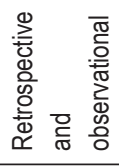 & 468 & 146 & 125 & * & $\begin{array}{l}\text { Bad COVID-19/ } \\
\text { hospitalisations in RA: } 79 \text {; } \\
\text { SpA: } 20\end{array}$ & $\begin{array}{l}\text { Older age } \\
\text { Concomitant diseases } \\
\text { Steroid treatment }>5 \mathrm{mg} / \text { day } \\
\text { Moderate and high disease activity }\end{array}$ \\
\hline Montero et al. & 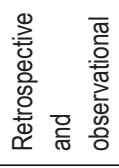 & 62 & 20 & 16 & * & $\begin{array}{l}\text { Bad COVID-19/ } \\
\text { hospitalisations in RA 15; } \\
\text { SpA: } 11 \\
\text { Deaths: } 10\end{array}$ & $\begin{array}{l}\text { Male gender } \\
\text { Pre-existing lung disease } \\
\text { Steroid treatment }>5 \mathrm{mg} / \text { day }\end{array}$ \\
\hline Pang et al. & 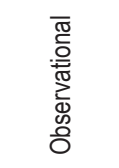 & 21 & 15 & - & - & $\begin{array}{l}\text { Bad COVID-19/ } \\
\text { hospitalisations in RA: } 15 \\
0 \text { deaths }\end{array}$ & Concomitant diseases \\
\hline Cheng et al. & 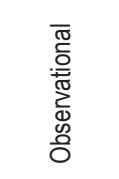 & 5 & 4 & - & - & $\begin{array}{l}5 \text { hospitalisations } \\
0 \text { deaths }\end{array}$ & Not reported \\
\hline Marques et al. & 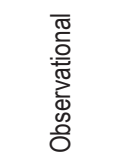 & 334 & 95 & 45 & 23 & $\begin{array}{l}110 \text { hospitalisations } \\
28 \text { deaths }\end{array}$ & $\begin{array}{l}\text { Age }>50 \text { years; Treatment with } \\
\text { steroids and cyclophosphamide }\end{array}$ \\
\hline
\end{tabular}

Legend: PsA: Psoriatic arthritis; RA: Rheumatoid arthritis; RMD; SpA: Spondyloarthritis

* In these studies, PsA was included in the SpA group

${ }^{* *}$ Adapted from [18] 
to the current situation is through the development of effective vaccines against SARS-CoV-2 [16]. Unfortunately, the lack of a common opinion on vaccination over the last two decades [20], as well as the „rapid“ development, production and approval of several COVID-19 vaccines, has led to public distrust and called into question the medical assessment in a pandemic. Documented adverse events - cases of thrombosis in young women under 50 years, have strengthened negative attitudes in society [21]. This fluctuation is exacerbated in patients with autoimmune rheumatic diseases, as they did not participate in the first phases of a clinical trial of COVID-19 vaccines [22].

In a study of 42 healthy individuals and 26 patients with chronic inflammatory diseases, mRNA vaccines were immunized and antibodies made against the virus were tested. Antibody titers were tested by ELISA before the first dose and 7 days after the second dose. Disease activity and adverse events were also observed 7 days before and after vaccination. The results showed that after the second dose of vaccine, neutralizing antibodies and total anti-SARS-CoV-2 lgG were observed in all patients with chronic inflammatory diseases, as well as in the general population group. Patients have slightly lower levels of neutralizing antibodies $-87.42 \%$, compared to the other group - 96.04\%. In the same study, they looked at the frequency and type of side effects after vaccination in patients with chronic inflammatory diseases (CID), compared to the general population. Mild systemic side effects such as fatigue and myalgia are more common in patients with CID than in healthy individuals $(53.8 \%$ vs. $43.2 \%$ and $42.3 \%$ vs. $31.6 \%$, respectively). The results are similar for headache as a side effect $(38.5 \%$ in CID versus $35.1 \%$ in healthy people). Fever was not observed in patients with CID, in contrast to $13.5 \%$ in healthy individuals. There was no exacerbation of the underlying disease in any of the patients during the study. No change in DMARDs treatment or corticosteroid dose was required in any of the patients with CID [23].

Two independent cohort studies with patients with immunomediated inflammatory diseases treated with methotrexate investigated the efficacy of COVID-19 vaccines in building a humoral and cellular immune response. The results showed that in healthy individuals and patients with ARD treated with bDMARDs (most often anti-TNF) a strong immune response was demonstrated (in over $90 \%$ of patients), while in patients treated with methotrexate an adequate antibody titer was barely achieved. at
$62.2 \%$. The results showed that patients with immunomediated inflammatory diseases did not show an increase in CD8 + T-cell activation after vaccination [24]. These results are also confirmed by a dermatological study conducted in the UK on humoral immunity after vaccination, which included patients with psoriasis on methotrexate therapy or biologic therapy (anti-TNF, anti-IL-17 or anti-IL-23). Data showed that functional humoral immunity after a single dose of BNT162b2 (Pfizer-BioNTech) is impaired by methotrexate but not by biological therapy [25].

A study in Switzerland looked at 53 patients with rheumatoid arthritis treated with DMARDs and 20 healthy individuals immunized against COVID-19 - the vaccines given were mRNA-1273 (Moderna) and BNT162b2 (Pfizer-BioNTech). Quantitative antibodies to SARS-CoV-2 spike protein 1 were tested three weeks after the first dose and two weeks after the second dose. Induced antibody titers were significantly lower in patients with rheumatoid arthritis, with $90 \%$ of the control group reaching high levels after the first dose, compared with $10 \%$ of patients with RA. Twelve percent of patients did not reach the required antibody titers after the second dose of the vaccine, and half of them received treatment with targeted synthetic DMARDs or biological DMARDs as monotherapy or combination therapy [26].

We are given a more detailed idea of the data from two studies conducted in Amsterdam, the Netherlands, in which all patients with systemic autoimmune diseases from the Amsterdam Rheumatology and Immunology Center and patients with multiple sclerosis from the Amsterdam Multiple sclerosis Center were invited. Each patient completed a questionnaire on whether they had been vaccinated against SARSCoV-2, whether they had a local or systemic adverse reaction during the first 7 days, and whether they had an exacerbation of the underlying disease within 2 months of vaccination. The study included 505 patients (of which 204 with RA and 81 with MS) and 203 healthy controls. ChAdOx1 nCoV-19 (AstraZeneca) and BNT162b2 (Pfizer/BioNTech) are the most common vaccines among patients. $258(51 \%)$ patients and 106 (52\%) healthy individuals had at least one mild side effect (without limiting daily activities), 105 (21\%) patients, and 38 (19\%) healthy subjects reported a moderate side effect. (limiting daily activities). Severe side effects (requiring medical attention) are very rare (6-1\% in patients and 0 in the control group), there were no serious side effects requiring hospitalization. The most common complaints after vaccination were pain at the injection site, fatigue, headache. Joint complaints were more common in patients 
with rheumatic diseases - $49(10 \%)$ than in healthy individuals $-3(1 \%)$, but only a small proportion of patients (26 [5\%]) reported an exacerbation of their autoimmune disease. up to 2 months after vaccination. The analysis of this study demonstrated that the side effects of COVID-19 vaccination in patients with autoimmune rheumatic diseases were comparable to the control group, regardless of the type of vaccine. Additionally, data suggest that COVID-19 vaccination does not exacerbate primary ARD [27].

In support of these data, a case of exacerbation of rheumatoid arthritis has been reported in the United States following vaccination with COVID-19-BNT162b2 (BioNTech-Pfizer). The patient was a 55-year-old white man with non-erosive seropositive rheumatoid arthritis (rheumatoid factor positive, anti-CCP, ANA and anti-Ro antibodies) who had been in clinical remission with upadacitinib monotherapy for over 2 years and exacerbated rheumatoid arthritis 12 hours after of the vaccine. Nine days after the second dose, the patient has significant swelling and warmth in the right knee, as well as pain in flexion and extension, increased CRP and ESR. In knee arthrocentesis, $24 \mathrm{ml}$ of synovial fluid was punctured, in which 24385 cells were found, of which $62 \%$ neutrophils, 30\% monocytes and $8 \%$ lymphocytes, without crystals and bacteria [29].

The literature reference gives us information about a case of IgA vasculitis with renal and gastrointestinal involvement in a patient in remission of 2 years, in whom an exacerbation of the condition was observed after vaccination with mRNA-1273 (Moderna). Seven days after vaccination, the patient developed acute diffuse abdominal pain and diarrhea (6 times daily) with increased inflammatory markers, evidence of sigmoid wall thickening with peripheral infiltration (from CT of the abdomen) and the appearance of palpable purpura on the lower extremities [30]. Two other articles describe three cases of exacerbation of $\lg$ A nephropathy after vaccination - with the appearance of macrohematuria and an increase in the amount of protein excreted in the urine. In one case, the proteinuria before vaccination was $0.63 \mathrm{~g} / \mathrm{d}$, while 3 weeks after the second dose it reached $1.40 \mathrm{~g} / \mathrm{d}$ $(31,32)$. Cohen SR et al. describes an exacerbation of leukocytoclastic vasculitis in a patient with a history of psoriasis, psoriatic arthritis, inflammatory bowel disease and vasculitis. The patient had no exacerbations in the last 2 years, but 2 days after the first dose of BNT162b2 mRNA (Pfizer-BioNTech) she noticed the appearance of palpable purpura on the lower extremities, which intensified after the second dose of the vaccine, covering the upper extremities, lower back and abdomen, without systemic manifestation [33].
Given the high efficacy of COVID-19 vaccines, the benefits of vaccinating vulnerable groups outweigh the risk of exacerbation of ARDs. This is the reason why experts from the American College of Rheumatology recommend vaccination in patients with autoimmune rheumatic diseases [29, 34, 35.

\section{CONCLUSION}

COVID-19 changed behavioral algorithms in patients with autoimmune rheumatic diseases. Science still needs additional information about the effect of the virus on the human body, environmental factors and concomitant diseases that affect the characteristics of the disease and the severity of the disease. The new mRNA vaccines against SARS-CoV-2 have improved the prognosis for a milder course in patients. Studies show that immunogenicity and efficacy are over $90 \%$ in the immunocompetent adult population. However, there are still no definite answers regarding patients with immune-mediated inflammatory diseases. The current data show that the risk factors for hospitalization and intensive care do not differ significantly in patients with ARD compared to the general population. Treatment with CS above $10 \mathrm{mg} /$ day, a large number of concomitant diseases remain a major risk factor in this group of patients. Methotrexate treatment was associated with poorer antibody synthesis after vaccination, which was also seen in patients treated with Rituximab. There are currently no definite data on more frequent adverse events following SARS-CoV-2 vaccine in patients with immune-mediated inflammatory diseases.

\section{Библиография / References}

1. Stoilov N, Boyadzhieva, V. SARS-CoV-2 and autoimmune rheumatic diseases. Rheumatology (Bulgaria). 2021p28, 4 (Feb. 2021), 36-44. DOI:https://doi.org/10.35465/28.4.2020.pp36-44.

2. Furer V, Rondaan C, Agmon-Levin N, et al. Point of view on the vaccination against COVID-19 in patients with autoimmune inflammatory rheumatic diseases. RMD Open. $2021 \mathrm{Feb} ; 7(1): \mathrm{e} 001594$.

3. Monti S, Balduzzi S, Delvino $P$, et al. Clinical course of COVID-19 in a series of patients with chronic arthritis treated with immunosuppressive targeted therapies. Ann Rheum Dis. 2020 May;79(5):667-668.

4. Tomelleri A, Sartorelli S, Campochiaro C, et al. Impact of COVID-19 pandemic on patients with large-vessel vasculitis in Italy: a monocentric survey. Ann Rheum Dis. 2020 Sep;79(9):1252-1253.

5. Bower H, Frisell T, Di Giuseppe D et al. ARTIS Study Group. Impact of the COVID-19 pandemic on morbidity and mortality in patients with inflammatory joint diseases and in the general population: a nationwide Swedish cohort study. Ann Rheum Dis. 2021:annrheumdis-2021-219845. 
6. Hasseli R, Mueller-Ladner U, Hoyer BF et al. Older age, comorbidity, glucocorticoid use and disease activity are risk factors for COVID-19 hospitalisation in patients with inflammatory rheumatic and musculoskeletal diseases. RMD Open. 2021;7:e001464.

7. D'Silva KM, Jorge A, Cohen A, et al. COVID-19 Outcomes in Patients With Systemic Autoimmune Rheumatic Diseases Compared to the General Population: A US Multicenter, Comparative Cohort Study. Arthritis Rheum. 2021 Jun;73(6):914-920.

8. Porfidia A, Valeriani E, Pola R, et al. Venous thromboembolism in patients with COVID-19: Systematic review and meta-analysis. Thromb Res. 2020 Dec;196:67-74.

9. Kim SC, Schneeweiss S, Liu J, Solomon DH. The risk of venous thromboembolism in patients with rheumatoid arthritis. Arthritis Care Res 2013;65:1600-7.

10. Strangfeld A, Schäfer M, Gianfrancesco MA, et al. Factors associated with COVID-19-related death in people with rheumatic diseases: results from the COVID-19 Global Rheumatology Alliance physician-reported registry. Ann Rheum Dis. $2021 \mathrm{Jul} ; 80(7): 930-942$.

11. Gianfrancesco M, Hyrich KL, Al-Adely S, et al. Characteristics associated with hospitalisation for COVID-19 in people with rheumatic disease: data from the COVID-19 Global Rheumatology Alliance physician-reported registry. Ann Rheum Dis. 2020 Jul;79(7):859-866.

12. Mathian A, Mahevas M, Rohmer J et al. Clinical course of coronavirus disease 2019 (COVID-19) in a series of 17 patients with systemic lupus erythematosus under long-term treatment with hydroxychloroquine. Ann Rheum Dis. 2020, http://dx.doi.org/10.1136/annrheumdis-2020-217566

13. Askanase AD, Khalili L, Buyon JP. Thoughts on COVID-19 and autoimmune diseases. Lupus Sci Med, 2020, 7; e000396; http://dx.doi.org/10.1136/lupus-2020-000396

14. Bachiller-Corral J, Boteanu A, Garcia-Villanueva MJ, et al. Risk of Severe COVID-19 Infection in Patients With Inflammatory Rheumatic Diseases. J Rheumatol. 2021 Jul;48(7):1098-1102.

15. Hasseli R, Mueller-Ladner U, Schmeiser T, et al. National registry for patients with inflammatory rheumatic diseases (IRD) infected with SARS-CoV-2 in Germany (ReCoVery): a valuable mean to gain rapid and reliable knowledge of the clinical course of SARS-CoV-2 infections in patients with IRD. RMD Open. 2020 Sep;6(2):e001332.

16. Marques CDL, Kakehasi AM et al. High levels of immunosuppression are related to unfavourable outcomes in hospitalised patients with rheumatic diseases and COVID-19: first results of ReumaCoV Brasil registry. RMD Open. 2021 Jan; 7(1):e001461.

17. Ruscitti $\mathrm{P}$, Conforti $\mathrm{A}$ et al. Pathogenic implications, incidence, and outcomes of COVID-19 in autoimmune inflammatory joint diseases and autoinflammatory disorders. Adv Rheumatol. $2021 \mathrm{Jul}$ 8;61(1):45.

18. COVID-19 Map-Johns Hopkins Coronavirus Resource Center. Johns Hopkins Coronavirus Resource Center. 2020. https: // coronavirus. Jhu.edu /map.html. Accessed on 26 JUL 2021

Постъпил за печат: 30.10.2021 2.

$\checkmark$ Адрес за кореспонденция:

Д-р Ж. Димова, дм

Клиника по ревматология

УМБАЛ "Св. Ив. Рилски"

MY - София
19. COVID-19 vaccines. 2020. https://www.who.int/emergencies/diseases/novel-coronavirus-2019/ covid19-vaccines. Accessed on 26 JUL 2021

20. Dubé E, Vivion M, MacDonald NE. Vaccine hesitancy, vaccine refusal and the anti-vaccine movement: influence, impact and implications. Expert Rev Vaccines. 2015 Jan;14(1):99-117

21. Trogen B, Oshinsky D, Caplan A. Adverse Consequences of Rushing a SARS-CoV-2 Vaccine: Implications for Public Trust. JAMA. 2020 Jun 23;323(24):2460-2461

22. Boekel L, Hooijberg F, et al. Perspective of patients with autoimmune diseases on COVID-19 vaccination. Lancet Rheumatol. 2021 Apr;3(4):e241-e243.

23. Geisen UM, Berner DK, Tran F. Immunogenicity and safety of anti-SARS-Cov-2 mRNA vaccines in patients with chronic inflammatory conditions and immunosuppressive therapy in a monocentric cohort. Ann Rheum Dis2021;0:1-6

24. Haberman RH, Herati RS et al. Methotrexate Hampers Immunogenicity to BNT162b2 mRNA COVID-19 Vaccine in Immune-Mediated Inflammatory Disease. medRxiv [Preprint]. 2021 May 12:2021.05.11.21256917.

25. Mahil SK, Bechman K et al. The effect of methotrexate and targeted immunosuppression on humoral and cellular immune responses to the COVID-19 vaccine BNT162b2: a cohort study. Lancet Rheumatol. 2021 Jul 8.

26. Rubbert-Roth A, Vuilleumier $\mathrm{N}$ et al. Anti-SARS-CoV-2 mRNA vaccine in patients with rheumatoid arthritis. Lancet Rheumatol. 2021 Jul;3(7):e470-e472.

27. Boekel L, Kummer LY et al. Adverse events after first COVID-19 vaccination in patients with autoimmune diseases. Lancet Rheumatol. 2021 Aug;3(8):e542-e545.

28. Felten $R$, Kawka $L$ et al. Tolerance of COVID-19 vaccination in patients with systemic lupus erythematosus: the international VACOLUP study. Lancet Rheumatol. 2021 Jul 21

29. Terracina KA, Tan FK. Flare of rheumatoid arthritis after COVID-19 vaccination. Lancet Rheumatol. $2021 \mathrm{Ju}$ l;3(7):e469-e470

30. Obeid M, Fenwick C, Pantaleo G. Reactivation of IgA vasculitis after COVID-19 vaccination. Lancet Rheumatol. 2021 Jul 6.

31. Negrea $L$, Rovin BH. Gross hematuria following vaccination for severe acute respiratory syndrome coronavirus 2 in 2 patients with IgA nephropathy. Kidney Int. 2021 Jun;99(6):1487.

32. Rahim SEG, Lin JT, Wang JC. A case of gross hematuria and IgA nephropathy flare-up following SARS-CoV-2 vaccination. Kidney Int. 2021 Jul;100(1):238.

33. Cohen $S R$, Prussick $L$ et al. Leukocytoclastic vasculitis flare following the COVID-19 vaccine. Int J Dermatol. 2021 Aug;60(8):1032-1033.

34. Polack FP, Thomas SJ, Kitchin N et al. Clinical Trial Group. Safety and Efficacy of the BNT162b2 mRNA Covid-19 Vaccine. N Engl J Med. 2020 Dec 31;383(27):2603-2615.

35. Mortezavi M, Menon S, Lee K, Rivas J. Use of tofacitinib in the context of COVID-19 vaccination: comment on the American College of Rheumatology clinical guidance for COVID-19 vaccination in patients with rheumatic and musculoskeletal diseases. Arthritis Rheumatol. 2021 May 27:10.1002/art.41806.

\section{Submitted: 30.10 .2021}

$\triangle$ Correspondence address:

Z. Dimova, MD

Clinic of Rheumatology

University Hospital "Sv. Iv. Rilski"

MU - Sofia 